ARTICLE

\title{
MOTS-c is an exercise-induced mitochondrial- encoded regulator of age-dependent physical decline and muscle homeostasis
}

Joseph C. Reynolds (10 1, Rochelle W. Lai ${ }^{1}$, Jonathan S. T. Woodhead ${ }^{2,3}$, James H. Joly ${ }^{4}$, Cameron J. Mitchell (1) 5,6, David Cameron-Smith ${ }^{5}$, Ryan Lu', Pinchas Cohen (1) 1,7, Nicholas A. Graham (1) 4,7, Bérénice A. Benayoun (1) 1,7,8, Troy L. Merry ${ }^{5,6}$ \& Changhan Lee (1) 1,7,9凶

Healthy aging can be promoted by enhanced metabolic fitness and physical capacity. Mitochondria are chief metabolic organelles with strong implications in aging that also coordinate broad physiological functions, in part, using peptides that are encoded within their independent genome. However, mitochondrial-encoded factors that actively regulate aging are unknown. Here, we report that mitochondrial-encoded MOTS-c can significantly enhance physical performance in young ( $2 \mathrm{mo}$.), middle-age ( $12 \mathrm{mo}$.), and old (22 mo.) mice. MOTS-c can regulate (i) nuclear genes, including those related to metabolism and proteostasis, (ii) skeletal muscle metabolism, and (iii) myoblast adaptation to metabolic stress. We provide evidence that late-life (23.5 mo.) initiated intermittent MOTS-c treatment (3x/week) can increase physical capacity and healthspan in mice. In humans, exercise induces endogenous MOTS-c expression in skeletal muscle and in circulation. Our data indicate that aging is regulated by genes encoded in both of our co-evolved mitochondrial and nuclear genomes.

\footnotetext{
${ }^{1}$ Leonard Davis School of Gerontology, University of Southern California, Los Angeles, CA 90089, USA. ${ }^{2}$ Discipline of Nutrition, Faculty of Medical and Health Sciences, The University of Auckland, Auckland, New Zealand. ${ }^{3}$ Maurice Wilkins Centre for Molecular Biodiscovery, The University of Auckland, Auckland, New Zealand. ${ }^{4}$ USC Mork Family Department of Chemical Engineering and Materials Science, Los Angeles, CA 90089, USA. ${ }^{5}$ Liggins Institute, The University of Auckland, Auckland, New Zealand. ${ }^{6}$ School of Kinesiology, University of British Colombia, Vancouver, BC, Canada V6T 1Z3. ${ }^{7}$ USC Norris Comprehensive Cancer Center, Los Angeles, CA 90089, USA. ${ }^{8}$ USC Stem Cell Initiative, Los Angeles, CA 90089, USA. ${ }^{9}$ Biomedical Science, Graduate School, Ajou University, Suwon 16499, Korea. ${ }^{凶}$ email: changhan.lee@usc.edu
} 
$\mathrm{T}$ he progressive loss of metabolic homeostasis is a hallmark of aging, which impedes parenchymal function and ultimately diminishes physical capacity ${ }^{1,2}$. In fact, aging is a leading risk factor for a myriad of non-communicable chronic diseases $^{3-9}$. Organismal fitness requires continuous adaptive cellular stress responses to the ever-shifting internal and external environment. Mitochondria not only produce the bulk of cellular energy, but also coordinate adaptive cellular homeostasis by dynamically communicating to the nucleus ${ }^{10}$ and other subcellular compartments ${ }^{11}$. Mitochondrial communication is mediated by multiple nuclear-encoded proteins, transient molecules, and mitochondrial metabolites ${ }^{12}$.

Mitochondria possess a distinct circular genome that has been traditionally known to host only 13 protein-coding genes. However, short open reading frames (sORFs) encoded in the mitochondrial genome have been recently identified. Such sORFs produce bioactive peptides, collectively referred to as mitochondrial-derived peptides (MDPs), with broad physiological functions ${ }^{13,14}$. MOTS-c (mitochondrial ORF of the 12S rRNA type-c) is an MDP that promotes metabolic homeostasis, in part, via $A M P K^{15,16}$ and by directly regulating adaptive nuclear gene expression following nuclear translocation ${ }^{17,18}$. MOTS-c expression is age-dependent and detected in multiple tissues, including skeletal muscle, and in circulation ${ }^{15,16,19}$, thus it has been dubbed a "mitochondrial hormone"16 or "mitokine"20,21. In fact, we previously reported that systemic MOTS-c treatment reversed diet-induced obesity and diet- and age-dependent insulin resistance in mice ${ }^{15}$. We tested if MOTS-c functions as a mitochondrial-encoded regulator of physical capacity and performance ${ }^{2,22,23}$ in young ( $2 \mathrm{mo}$.), middle-aged (12 mo.), and old $(22 \mathrm{mo}$.) mice.

Here, we show that exercise induces mtDNA-encoded MOTS-c expression in humans. MOTS-c treatment significantly (i) improves physical performance in young, middle-age, and old mice, (ii) regulates skeletal muscle metabolism and gene expression, and (iii) enhances adaptation to metabolic stress in $\mathrm{C} 2 \mathrm{C} 12$ cells in an HSF1-dependent manner.

\section{Results}

Endogenous MOTS-c levels increase upon exercise in humans. To determine if endogenous MOTS-c responds to physical exertion, and thus may be involved in driving adaptation to enhance physical capacity, we collected skeletal muscle and plasma from sedentary healthy young male volunteers $(24.5 \pm 3.7$ years old and BMI $24.1 \pm 2.1)$ that exercised on a stationary bicycle (Fig. 1a). Samples were collected before, during (plasma only), and after exercise and following a 4-h rest. Western blotting for endogenous MOTS-c in skeletal muscle revealed that relative levels (i.e. individual changes based on pre-exercise values) significantly increased after exercise (11.9-fold) and remained elevated after a 4 -h rest, albeit exhibiting a trend to return to baseline (Fig. 1b, c); see Supplementary Fig. 1a for absolute quantification values. ELISA revealed that relative levels of circulating endogenous MOTS-c also significantly increased during (1.6-fold) and after (1.5-fold) exercise, which then returned to baseline after $4 \mathrm{~h}$ of resting (Fig. 1d and Supplementary Fig. 1b). These findings suggest that exercise induces the expression of mitochondrial-encoded regulatory peptides in humans.

MOTS-c treatment improves physical performance in young mice. We next probed if MOTS-c functions as an exerciseinduced mitochondrial signal that improves physical capacity by treating young mice (CD-1; outbred) daily with MOTS-c [5 mg/ $\mathrm{kg} /$ day; intraperitoneal injections (IP)] for 2 weeks; we previously reported that 7 days of MOTS-c treatment improved skeletal muscle insulin sensitivity in young and older C57BL/6 mice $^{15}$. The rotarod performance test, whereby mice are placed on a rotating rod, revealed that daily MOTS-c significantly improved physical capacity and/or motor coordination (Supplementary Fig. 2a), but not grip strength (Supplementary Fig. 2b) in young mice. Because the rotarod test can also be affected by cognitive capacity, we assessed learning and memory using the Barnes maze and found no improvement (Supplementary Fig. 2c, d).

A treadmill running test confirmed that MOTS-c treatment can enhance physical performance. Because MOTS-c is a regulator of metabolic homeostasis that prevented high-fat diet (HFD)induced obesity and insulin resistance ${ }^{15}$, we tested if MOTS-c also improved running performance under metabolic (dietary) stress. We fed young mice (CD-1) a HFD (60\% calories from fat) and treated them with 2 doses of MOTS-c ( 5 and $15 \mathrm{mg} / \mathrm{kg} /$ day; IP; Supplementary Fig. 3a). Mice on the higher dose of MOTS-c showed significantly superior running capacity (Fig. 2a-c) and power output (joules; Supplementary Fig. 4a) following 10 days of treatment, but not following 7 days of treatment (Supplementary Fig. 5a). Notably, 7 days of MOTS-c treatment significantly improved skeletal muscle insulin sensitivity in young and older C57BL/6J mice also fed a high-fat diet ${ }^{15}$. We progressively increased the treadmill speed to test both endurance and speed. The final stage, which required mice to sprint $(23 \mathrm{~m} / \mathrm{min})$, was reached by $100 \%$ of mice on the higher dose of MOTS-c, but only $16.6 \%$ in the lower dose and control (vehicle) groups (Fig. 2d). Body composition analysis using a time-domain NMR analyzer revealed that both doses of MOTS-c significantly retarded fat gain and that the high dose significantly increased lean mass in young mice (CD-1; Supplementary Fig. 6a-c), in accord with prior reports ${ }^{15}$. There was no correlation between body weight and running time (Supplementary Fig. 7a). Taken together, performance improvements in treadmill running and rotarod test, paired with lack of changes in the grip strength and Barnes Maze, indicate an enhancement in physical capacity.

In young CD-1 mice, we simultaneously initiated MOTS-c treatment and a HFD (Supplementary Fig. 3a). To test if MOTS-c can improve physical performance in mice that have been on a
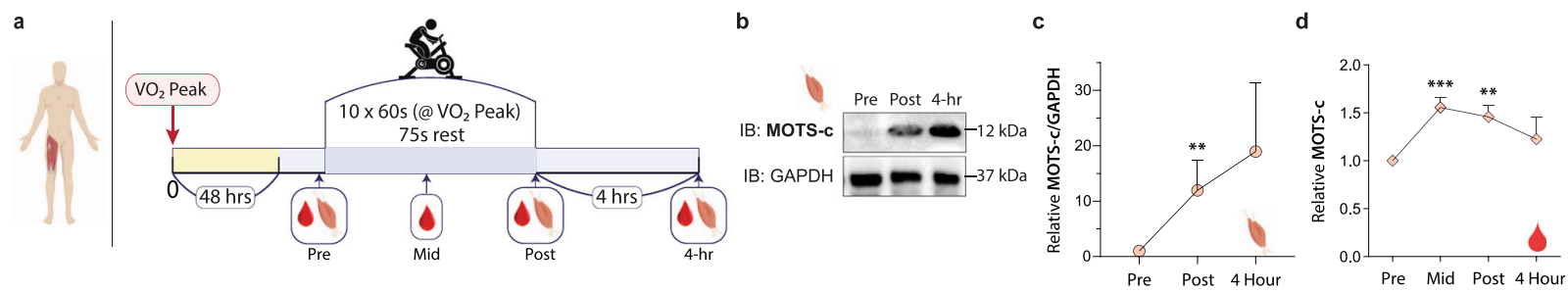

Fig. 1 MOTS-c responds to and regulates exercise in young subjects. a Schedule of exercise on a stationary bicycle and blood and skeletal muscle collection in young male subjects $(n=10)$. b, c Representative western blot of MOTS-c from skeletal muscle and quantification $(n=10 ; P=0.0098)$. $\mathbf{d}$ Quantification of serum MOTS-c levels by ELISA ( $n=10 ; P=0.0011 ; P=0.0021$, respectively). Data expressed as mean \pm SEM. Wilcoxon matched-pairs two-sided signed rank test was used for $(\mathbf{c}, \mathbf{d})$. 

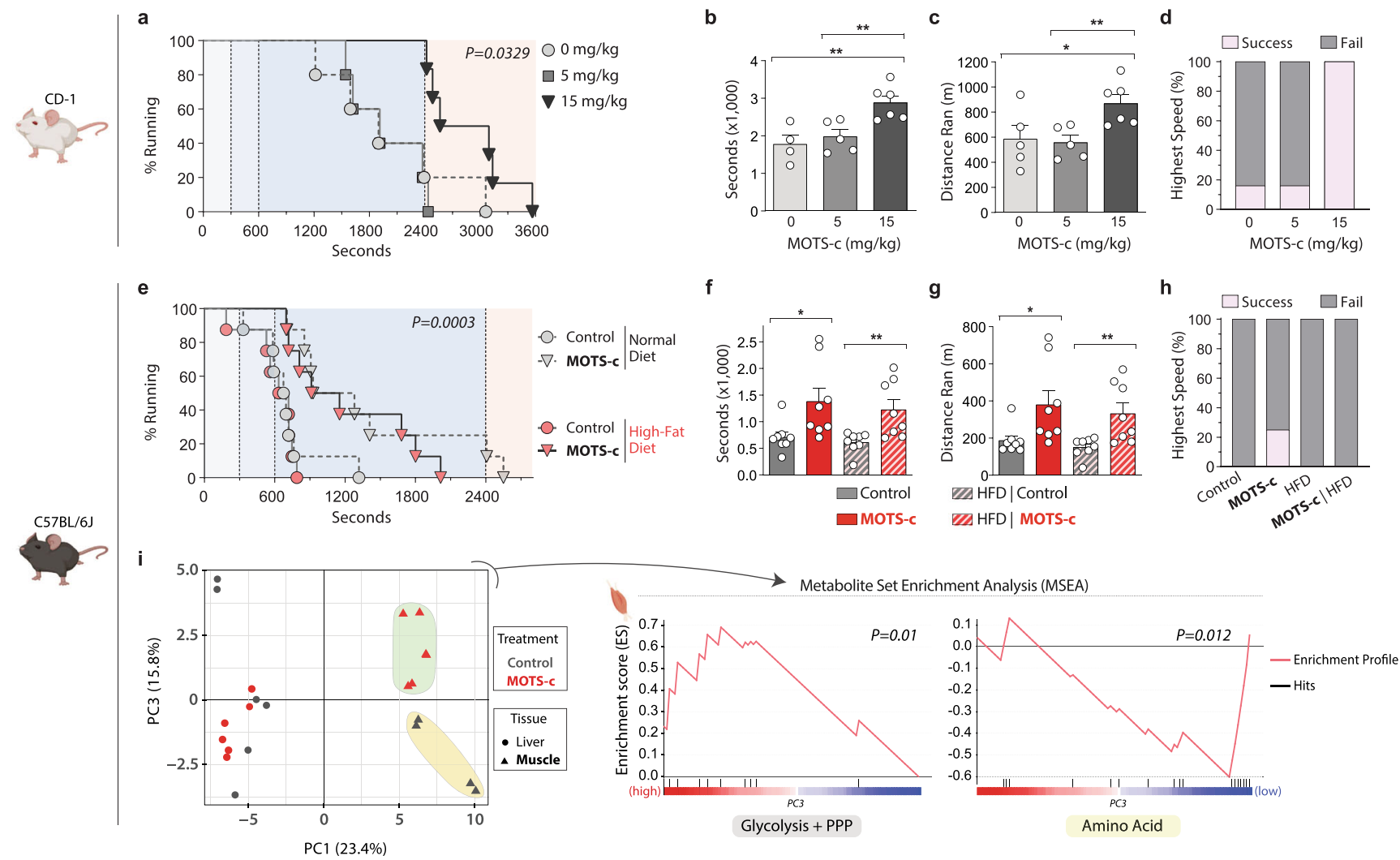

Fig. 2 MOTS-c treatment increases physical capacity in young mice regardless of diet. a-c Treadmill performance of 12-week-old male CD-1 (outbred) mice fed a normal diet ( $n=5$, Control and $5 \mathrm{mg} / \mathrm{kg}$ MOTS-c; $n=6,15 \mathrm{mg} / \mathrm{kg}$ MOTS-c); a running curves $(P=0.0329)$, $\mathbf{b}$ total time on treadmill $(P=$ $0.0069,0$ vs. $15 ; P=0.0081,5$ vs. 15$)$, c total distance ran $(P=0.0418,0$ vs. $15 ; P=0.0081,5$ vs.15), and $\mathbf{d}$ percent capable of reaching the highest speed (sprint). e-h Treadmill performance of 12-week-old male C57BL/6J (inbred) mice fed a HFD $(n=8)$; e running curves $(P=0.0003)$, $\mathbf{f}$ total time on treadmill ( $P=0.0237$, normal diet control vs. normal diet + MOTS-c; $P=0.0084$, HFD control vs. HFD + MOTS-c) $\mathbf{g}$ total distance ran $(P=0.0326$, normal diet control vs. normal diet + MOTS-c; $P=0.0087$, HFD Control vs. HFD + MOTS-c) h percent capable of reaching the highest speed (final stage). i PCA and MSEA on metabolomic data from skeletal muscle and liver of C57BL/6J mice that were fed a HFD, treated with MOTS-c, and exercised. Data expressed as mean \pm SEM. Log-rank (Mantel-Cox) test was used for a, e. Otherwise, all statistics were performed using the two-sided Student's $t$ test. ${ }^{\star} P<$ $0.05,{ }^{\star \star} P<0.01,{ }^{\star \star \star} P<0.001$.

HFD, we fed young C57BL/6J mice a HFD, or a normal diet, for 2 weeks before initiating daily MOTS-c injections $(15 \mathrm{mg} / \mathrm{kg} /$ day) for 2 weeks prior to a treadmill running test (Supplementary Fig. 3b). MOTS-c treatment significantly enhanced running performance and power output (joules; Supplementary Fig. 4b) on the treadmill regardless of the diet (Fig. 2e-h and Supplementary Fig. 5b). MOTS-c treatment enabled $25 \%$ of the young C57BL/6J mice to enter the final running stage (highest speed) on a normal diet, but none on a HFD (Fig. 2h). Consistent with our prior study ${ }^{15}$, MOTS-c treatment curbed HFD-induced weight gain in C57BL/6J mice (Supplementary Fig. 6d), which was largely driven by reduced fat accumulation (Supplementary Fig. 6e), but not loss of lean mass (Supplementary Fig. 6f), as determined by an NMR-based body composition analysis. However, there was no correlation between body weight and running time (Supplementary Fig. 7b). Also, consistent with our prior study ${ }^{15}$, there were no differences in food intake between control diet or HFD groups (Supplementary Fig. 8). Further, targeted metabolomics revealed that MOTS-c treatment significantly regulated (i) glycolysis/PPP (pentose phosphate pathway) and (ii) amino acid metabolism (Fig. 2i; Supplementary Figs. 3b and 9a; and Supplementary Data 1 and 2) in skeletal muscle, but not in liver, consistent with our previous study ${ }^{15}$. Together, these data indicate that MOTS-c treatment can improve overall physical performance, in part, by targeting skeletal muscle metabolism in young mice.
MOTS-c treatment enhances physical capacity in old mice. Aging is accompanied by a progressive decline in mitochondrial function ${ }^{1,24}$ and loss of metabolic homeostasis, in which MOTS-c may play a role ${ }^{2,10}$. Aging is associated with reduced MOTS-c levels in certain tissues, including the skeletal muscle, and in circulation ${ }^{15,19}$. We previously showed that an acute one-week MOTS-c treatment reversed age-dependent insulin resistance in mouse skeletal muscle ${ }^{15}$. Thus, we investigated if promoting metabolic homeostasis by MOTS-c treatment could reverse agedependent decline in physical capacity. Middle-aged (12 mo.) and old $(22 \mathrm{mo}$.) C57BL/6N mice were treated daily with MOTS-c $(15 \mathrm{mg} / \mathrm{kg} /$ day; IP) for 2 weeks, then subjected to a treadmill running test (Fig. 3a). Both middle-aged and old mice ran significantly longer with increased power output (joules) following MOTS-c treatment (Fig. 3b and Supplementary Fig. 4c). Old mice ran longer (2-fold; Fig. 3c) and farther (2.16-fold; Fig. 3d) when treated with MOTS-c. Further, MOTS-c enabled $17 \%$ of the old mice to enter the final running stage (highest speed), whereas none in the untreated group were successful (Fig. 3e). Notably, MOTS-c treatment enabled old mice to outperform untreated middle-aged mice, suggesting a more pervasive physical reprogramming rather than just rejuvenation. Respiratory exchange ratio (RER), measured using a metabolic cage, indicates fuel preference (1.0: carbohydrates, 0.7: fat). "Metabolic flexibility", which refers to the overall adaptive capacity to a shift in metabolic supply-demand equilibrium (e.g. exercise), declines with age ${ }^{25,26}$. 
a

Fig. 3

Fig. 4

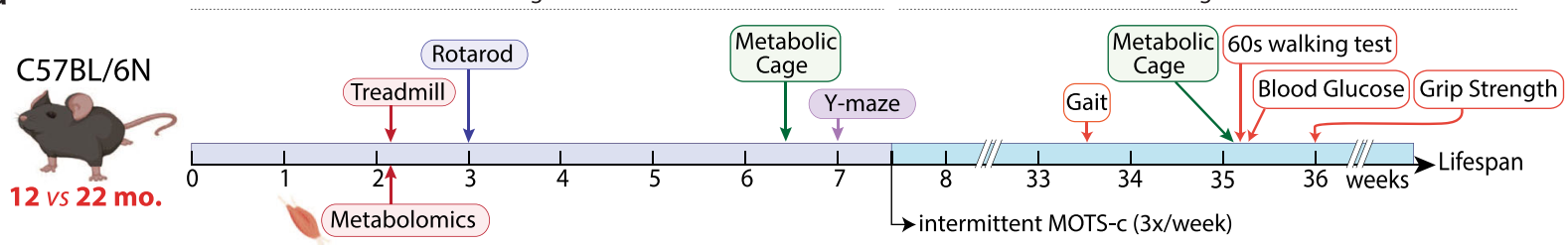

b

f
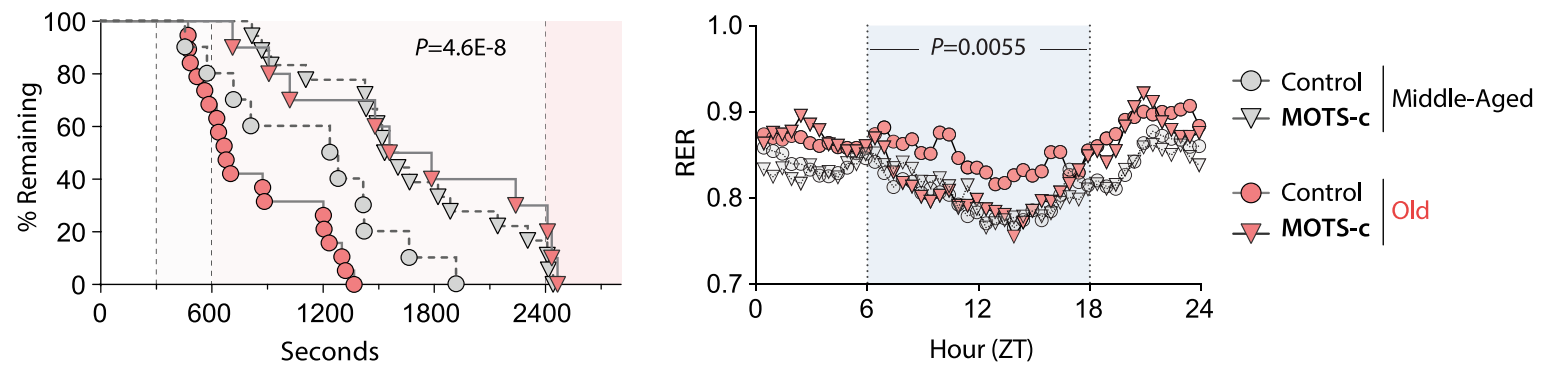

C

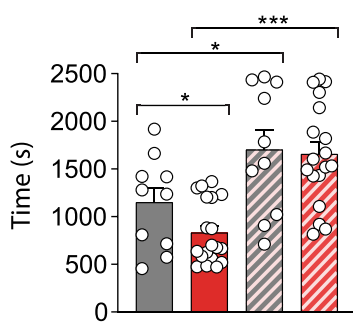

d

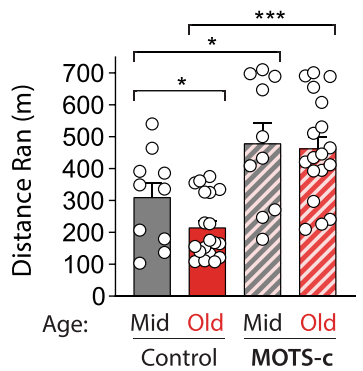

e

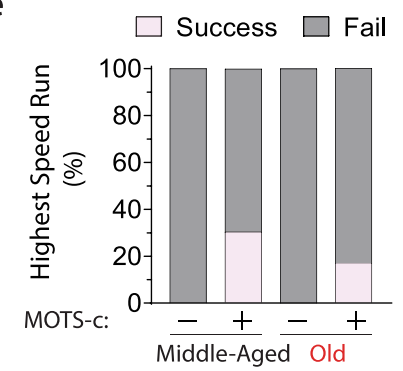

g

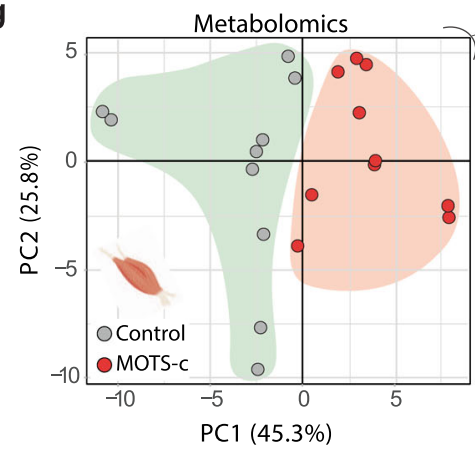

h

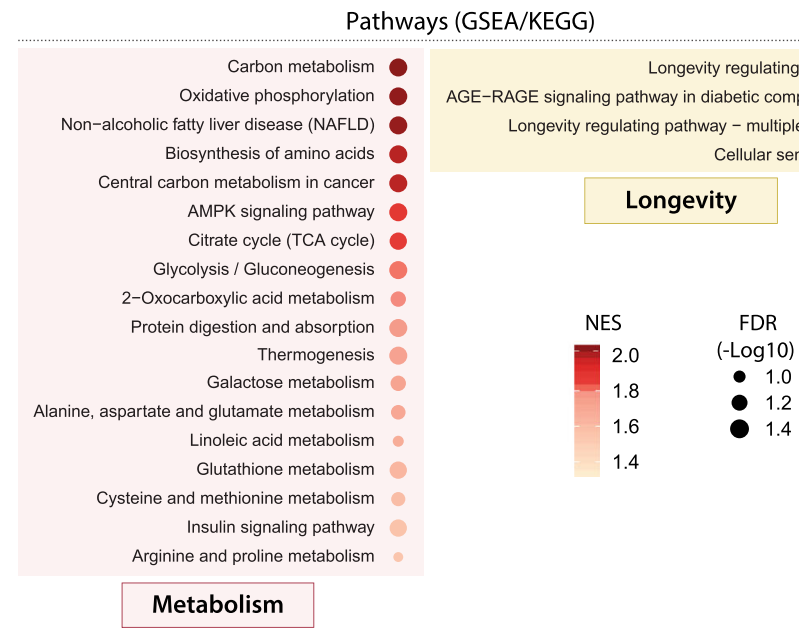

Fig. 3 Acute MOTS-c treatment enhances physical capacity in old mice. a Schedule of MOTS-c treatment and assays in middle-aged and old C57BL/ $6 \mathrm{~N}$ mice $(n=10$, MA groups; $n=19$, old control; $n=18$, old MOTS-c), including $\mathbf{b}$ treadmill running curves $(P=0.000000046)$, $\mathbf{c}$ total time on treadmill ( $P=0.0458$, MA control vs. old control; $P=0.0497$, MA control vs. MA MOTS-c; $P=0.000002$, old control vs. old MOTS-c), $\mathbf{d}$ total distance ran on treadmill ( $n=19$, old control; $n=18$, Old MOTS-c; $P=0.0464$, MA control vs. old control; $P=0.0462$, MA control vs. MA MOTS-c; $P=0.000002$, old control vs. old MOTS-c), and e percent capable of reaching the highest speed on a treadmill (final stage). $\mathbf{f}$ Respiratory exchange ratio (RER) following 2 weeks of daily MOTS-c injection $(n=4 ; P=0.0055)$. $\mathbf{g}$, $\mathbf{h}$ Skeletal muscle from treadmill-exercised old mice (22.5 months) treated daily with MOTS-c $(15 \mathrm{mg} / \mathrm{kg} /$ day) for 2 weeks $(n=10)$ were subject to $\mathbf{g}$ metabolomics and analyzed using PCA and MSEA and $\mathbf{h}$ GSEA analysis of muscle RNA-seq analysis. Balloon plots of select enriched terms using Gene Ontology Biological Process (GO_BP) database at false discovery rate $(F D R)<15 \%$. NES Normalized Enrichment Score. Full GSEA results are available in Supplementary Data 5. Data expressed as mean \pm SEM. Log-rank (Mantel-Cox) test was used for $\mathbf{b}$ and two-way ANOVA (repeated measures) was used for $\mathbf{f}$. GSEA statistics from R package 'clusterProfiler' were used for $\mathbf{h}$. Otherwise, all statistics were performed using the two-sided Student's $t$ test. ${ }^{\star} P<0.05,{ }^{\star \star} P<0.01$, ${ }^{\star \star \star} P<0.001$. MA middle-age. 
Indeed, we observed a shift in RER between daytime and nighttime, which trended more towards statistical significance for middle-aged mice $(P=0.076)$ compared to aged mice $(P=0.39$; Fig. 3f and Supplementary Fig. 10), indicating reduced metabolic flexibility with age. RER during daytime, but not nighttime, was significantly different between middle-aged vs. old mice, whereby old mice preferred to continue to utilize carbohydrates more than their middle-aged counterparts (Fig. 3f and Supplementary Fig. 10). This phenomena was fully reversed by MOTS-c treatment and the RER of old mice exhibited a similar circadian pattern to that of middle-aged mice (Fig. $3 \mathrm{f}$ and Supplementary Fig. 10). Such circadian-dependent effects of MOTS-c RER may be affected by food intake, as mice feed mostly during the night (Supplementary Fig. 11). There was no correlation between body weight and running time upon MOTS-c treatment (Supplementary Fig. 7c). Metabolomic analysis on skeletal muscle collected immediately post-exercise (a 30 -min run at a fixed moderate speed) in MOTS-c-treated (2 weeks) mice revealed that MOTS-c significantly regulated glycolysis and amino acid metabolism (Fig. 3g, Supplementary Fig. 9b, and Supplementary Data 3 and 4); the skeletal muscles of non-exercised mice did not show significant alterations in response to MOTS-c (Supplementary Fig. 12), suggesting that MOTS-c induces an adaptive metabolic response to exercise. To begin to understand the molecular mechanisms underlying the effects of MOTS-c, we performed RNA-seq analysis on the same skeletal muscles used for metabolomics. Although individual-to-individual variability was high, Gene Set Enrichment Analysis (GSEA) using the KEGG pathway database revealed that MOTS-c regulated processes related to (i) metabolism, including those known to be regulated by MOTS-c (e.g. AMPK signaling, glycolysis, and central carbon metabolism ${ }^{15,17}$, and (ii) longevity (FDR $<15 \%$; select pathways in Fig. $3 \mathrm{~h}$; full DESeq2 analysis in Supplementary Data 5 and full GSEA analysis in Supplementary Data 6). Gene Ontology Biological Process (GO_BP) analysis revealed a broader range of processes, including metabolism (lipid, carbohydrate, amino acid, and nucleotides), oxidative stress response, immune response, and nuclear transport (FDR $<15 \%$; select pathways in Supplementary Fig. 13; full analysis in Supplementary Data 6), again, consistent with our previous studies ${ }^{15,17}$. The rotarod performance test reinforced that MOTS-c treatment improved physical capacity in old mice (Supplementary Fig. 14a), while learning and memory was not affected as determined using the Y-maze test (Supplementary Fig. 14b), consistent with our observations in young mice (Supplementary Fig. 2). Together, these data suggest that MOTS-c treatment can significantly improve physical capacity in old mice, in part, by regulating skeletal function and improving "metabolic flexibility".

Late-life MOTS-c treatment improves mouse healthspan. Antiaging interventions that are applied later in life would be more translationally feasible compared to life-long treatments ${ }^{27-29}$. Building on the treadmill running tests, we tested if a late-life initiated ( $\sim 24$ mo.) intermittent (LLII) MOTS-c treatment ( $\overline{3} \mathrm{x} /$ week; $15 \mathrm{mg} / \mathrm{kg} /$ day) would improve healthy lifespan (Fig. 3a). To assess healthspan, towards the end-of-life (>30 mo.), we performed a battery of physical tests to further probe the effect of MOTS-c on reversing age-dependent physical decline (Fig. 3a). LLII MOTS-c improved (i) grip strength (Fig. 4a), (ii) gait, assessed by stride length (Fig. 4b), and (iii) physical performance, assessed by a 60 -second walking test (running was not possible at this age; Fig. 4c). In humans, reduced stride length and walking capacity are strongly linked to mortality and morbidity ${ }^{30}$. Together, these data indicate that LLII MOTS-c treatment improves physical capacity in old mice.
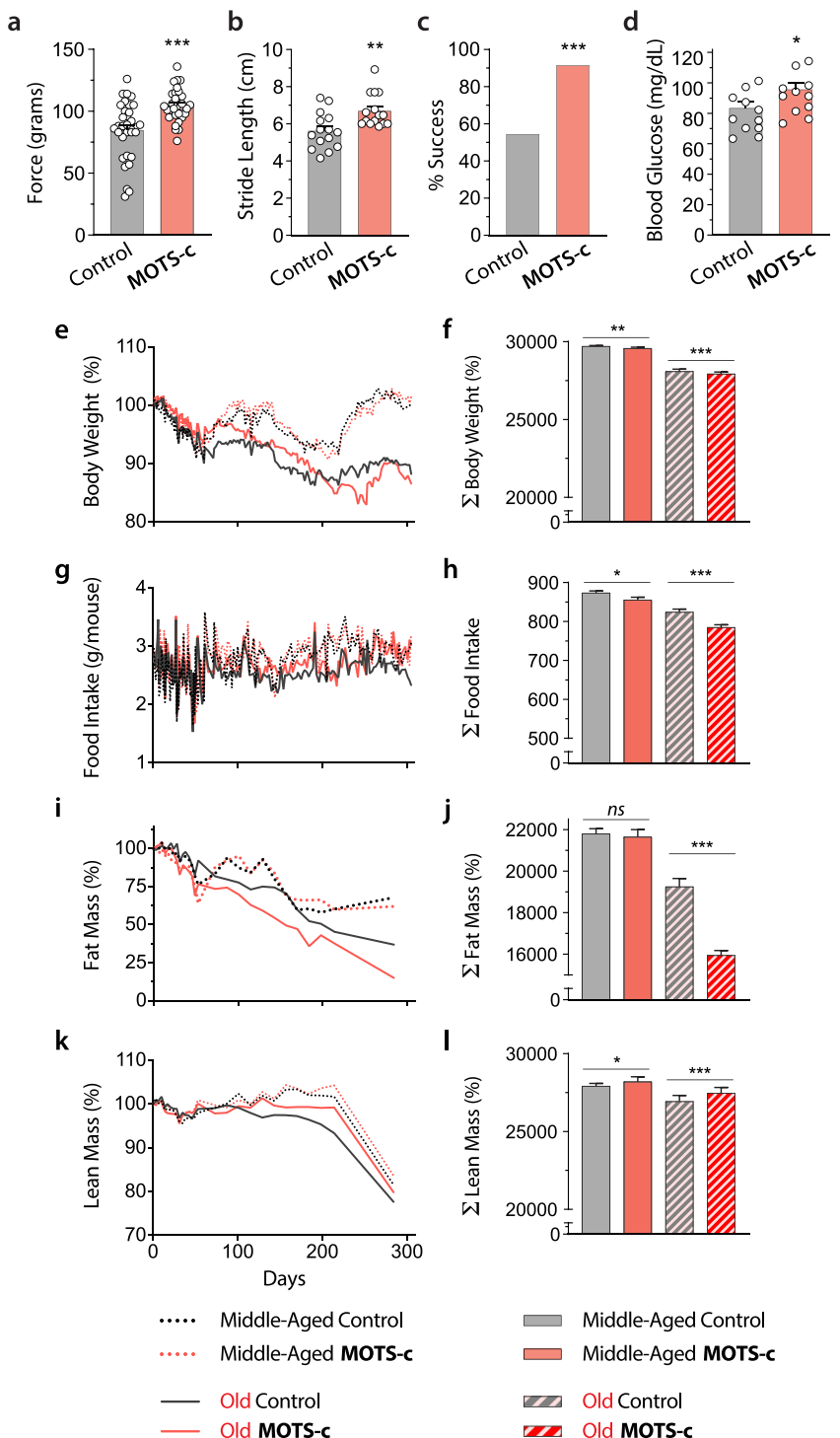

m

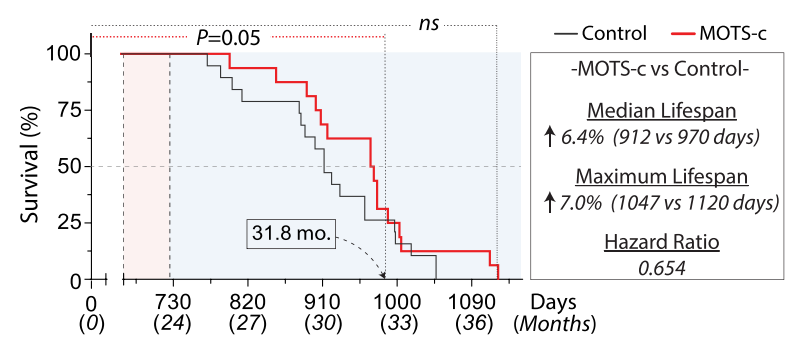

Independent lines of research have shown that MOTS-c is a mitochondrial-encoded metabolic regulator at the cellular and organismal level ${ }^{15,17,19,31-35}$. We posited that LLII MOTS-c treatment would cause metabolic reprogramming in old mice. Consistent with our previous report ${ }^{15}$, non-fasting blood glucose was better maintained in LLII MOTS-c-treated old mice $(30 \mathrm{mo}$; Fig. 4d). Over course of their life, LLII MOTS-c-treated mice showed comparable body weight to their untreated counterparts (Fig. 4e, f). However, total food intake was significantly reduced (Fig. 4g, $\mathrm{h}$ and Supplementary Fig. 11), whereas total activity was significantly higher (Supplementary Fig. 15). Yet, the activity changes at 30 months of age was marginal and may not account for considerable increase in energy expenditure that could explain the effect of MOTS-c on body weight at different age groups 
Fig. 4 MOTS-c regulates aging metabolism and healthspan. Life-long measurements on male C57BL6/N mice treated intermittently (3x/week) with MOTS-c ( $15 \mathrm{mg} / \mathrm{kg} /$ day) starting at middle and old age (13.5 and 23.5 mo.) as described in Fig. 2a. a grip strength test ( $n=11$; run in triplicate; $P=0.000078$ ), $\mathbf{b}$ gait analysis (stride length; $n=5$; run in triplicate; $P=$ 0.0038 ), c 60 -s walking test ( $n=11$, control; $n=12$, MOTS-c; $P=0.0428$ ), and $\mathbf{d}$ blood glucose levels $(n=11 ; P=0.0397)$. e, $\mathbf{f}$ Body weight $\mathbf{e}$ as a function of time and $\mathbf{f}$ the total sum ( $\sum ; n=19$, old control; $n=18$, old MOTS-c; $P=0.0013$, MA control vs. MA MOTS-c; $P=0.001$, old control vs. old MOTS-c); $\mathbf{g}$, $\mathbf{h}$ Food intake $\mathbf{g}$ as a function of time and $\mathbf{h}$ the total $\operatorname{sum}\left(\sum ; n=19\right.$, old control; $n=18$, old MOTS-c; $P=0.0276$, MA control vs. MA MOTS-c; $P=0.00005$, old control vs. old MOTS-c); $\mathbf{i}$, j Percent fat mass $\mathbf{i}$ as a function of time and $\mathbf{j}$ the total sum $\left(\sum ; n=19\right.$, old control; $n=$ 18, old MOTS-c; $P<1 \mathrm{E}-50)$; $\mathbf{k}$, I Percent lean mass $\mathbf{k}$ as a function of time and $\mathbf{I}$ the total sum $\left(\sum ; P=0.026\right.$, MA control vs. MA MOTS-c; $P=$ 0.0003 , old control vs. old MOTS-c). $\mathbf{m}$ Lifespan curve; $P=0.05$ until 31.8 months of age. Overall curve trended towards increased median and maximum lifespan $(P=0.23)$. Data expressed as mean $\pm \mathrm{SEM}$. Log-rank (Mantel-Cox) test was used for $\mathbf{m}$. Otherwise, all statistics were performed using the two-sided Student's $t$ test. ${ }^{\star} P<0.05,{ }^{\star \star} P<0.01,{ }^{\star \star \star} P<0.001$. MA middle-age.

(Supplementary Fig. 15). A likely possibility that could increase energy expenditure is, consistent with previous reports from our lab and others ${ }^{15,36}$, increased heat production. Body composition analysis using a time-domain NMR analyzer revealed significant reduction of fat mass (Fig. $4 \mathrm{i}, \mathrm{j}$ ) and a modest reduction in agedependent loss of lean mass (Fig. 4k, l). The RER, measured using metabolic cages, at $30 \mathrm{mo}$. revealed increased fat utilization, consistent with that obtained at $\sim 23.5 \mathrm{mo}$. (Fig. $3 \mathrm{f}$ ), but with a circadian shift compared to the same mice measured 6 months before (Supplementary Fig. 16); this is also consistent with reduced total fat mass (Fig. 4i, j and Supplementary Fig. 6b, e) and increased lipid utilization ${ }^{15,35}$. Ultimately, LLII MOTS-c treatment showed a trend towards increased median (6.4\%) and maximum $(7.0 \%)$ lifespan and reduced hazard ratio $(0.654) ; P=$ 0.05 until 31.8 months (Fig. $4 \mathrm{~m}$ ). Larger cohorts will be needed to confirm the broader significance of MOTS-c treatment on overall longevity. These data suggest that LLII MOTS-c treatment improves overall physical capacity in old mice and may compress morbidity and increase healthspan.

MOTS-c promotes metabolic adaptations in vitro. Skeletal muscle must adapt to various exercise-induced challenges ${ }^{37}$, including nutrient (e.g. metabolic supply-demand imbalance $)^{38}$, oxidative $^{39,40}$, and heat stress 37,41 , which share mitochondria as a common denominator. Because MOTS-c enhanced cellular resistance against metabolic/oxidative stress ${ }^{17}$, we tested if MOTS-c treatment improved skeletal muscle adaptation to metabolic stress using C2C12 mouse myoblast cells. Using crystal violet staining to determine cellular viability, we found that MOTS-c $(10 \mu \mathrm{M})$ treatment significantly protected C2C12 cells ( 2-fold) from $48 \mathrm{~h}$ of metabolic stress [glucose restriction (GR; $0.5 \mathrm{~g} / \mathrm{L}$ ) and serum deprivation (SD; $1 \%$ FBS)] (Fig. 5a). Next, we tested the replicative capacity of $\mathrm{C} 2 \mathrm{C} 12$ cells following prolonged metabolic stress as a functional marker of protection. C2C12 cells were metabolically stressed (GR/SD) for one week with daily MOTS-c $(10 \mu \mathrm{M})$ treatment, then replenished with complete medium for 2 days and stained with crystal violet. MOTS-ctreated $\mathrm{C} 2 \mathrm{C} 12$ cells showed significantly enhanced proliferative capacity within 2 days ( 6-fold; Fig. 5b). Because MOTS-c promotes fat utilization, which may underly its effect on "metabolic flexibility" (Figs. 3f and 4i, j and Supplementary Fig. 6) "15,35,42, we tested if MOTS-c-treated $\mathrm{C} 2 \mathrm{C} 12$ cells could survive on lipids without glucose $(0 \mathrm{~g} / \mathrm{L})$. As expected, most control cells died without glucose even with lipid supplementation, whereas MOTS-c treatment provided significant protection ( 2-fold; Fig. 5c). Real-time metabolic flux analysis revealed that MOTS-c treatment significantly increased lipid utilization capacity through increasing OCR, but not ECAR, in the presence of palmitate in C2C12 cells (Fig. 5d and Supplementary Fig. 17).

We previously reported that endogenous MOTS-c translocates to the nucleus to directly regulate adaptive nuclear gene expression in response to cellular stress ${ }^{17}$. Using fluorescently labeled MOTS-c peptide (MOTS-c-FITC), we confirmed that exogenously treated MOTS-c also dynamically translocated to the nucleus in a time-dependent manner (Fig. 5e $)^{17}$, indicating a direct nuclear role. We performed RNA-seq on $\mathrm{C} 2 \mathrm{C} 12$ cells treated with MOTS-c or vehicle control $(10 \mu \mathrm{M})$ under GR/SD for $48 \mathrm{~h}$ and found (i) separation of groups in PC1 (first principle component) using a principal component analysis (PCA; Fig. 5f) and (ii) 69 genes that were differentially regulated at FDR $<5 \%$ (Fig. 5g). Further, using the STRING (Search Tool for the Retrieval of Interacting Genes/Proteins) database to assess putative changes in protein-protein interaction networks based on our RNA-seq results, we found that a cluster related to heatshock responses, including Hsp40 (DNAJ) and Hsp70s (HSPA), were prominently regulated by MOTS-c in $\mathrm{C} 2 \mathrm{C} 12$ cells under GR/SD; we also identified previously reported MOTS-c targets, including Atf3, Jun, Fosl1, and Mafg ${ }^{17}$ (Fig. 5h and Supplementary Data 5). Indeed, select GO_BP analysis revealed that protein homeostasis (i.e. proteostasis) processes were significantly targeted with protein folding being the most significantly affected pathway in metabolically stressed myoblasts (Supplementary Fig. 18). Next, to identify common pathways in in vitro and in vivo models, we overlaid RNA-seq data from MOTS-c-treated (i) old mouse skeletal muscle and (ii) metabolically stressed (GR/ SD) C2C12. Consistently, select GO_BP analysis revealed several commonly targeted pathways by MOTS-c in both exercised skeletal muscle and metabolically stressed myoblasts that were related to proteostasis, including protein regulation and protein metabolism (select terms in Fig. 5i; full results in Supplementary Data 5 and 8); notably, commonly targeted pathways also provided a specific cellular context that reflects metabolic stress, including oxidative stress, mitochondria, cellular metabolism. To identify putative transcription factors involved in differential gene regulation upon MOTS-c treatment, we performed a statistical enrichment analysis inferred from the ChEA knowledge base ${ }^{43}$ $($ FDR $<5 \%)$. Consistent with the STRING and GSEA (GO_BP) analyses, we identified heat shock factor 1 (HSF1) as a putative transcription factor that was significantly enriched in both mouse skeletal muscle and myoblasts and could potentially regulate gene expression upon MOTS-c treatment (Fig. 5j). HSF1 is a master nutrient sensor and regulator of stress adaptation and proteostasis, in part, by inducing the expression of a myriad of heat shock proteins, including Hsp40 (DNAJ) and Hsp70s (HSPA), consistent with the effects of MOTS-c (Fig. 5h) ${ }^{44,45}$. Exercise induces HSF1 activation in skeletal muscle ${ }^{46}$ and cardiac muscle ${ }^{47}$ in rodents. Indeed, siRNA-mediated HSF1 knockdown reversed the protective effects of MOTS-c against GR/SD in myoblasts (Fig. 5k, 1). Together, these data suggest that MOTS-c improves metabolic homeostasis/flexibility and protein homeostasis in skeletal muscle under exercise-induced stress conditions.

\section{Discussion}

We previously reported that MOTS-c prevented diet-induced obesity and insulin resistance and reversed age-dependent skeletal muscle insulin resistance in $\mathrm{C} 57 \mathrm{BL} / 6 \mathrm{~J}$ mice, indicating a role in metabolic conditions such as diabetes ${ }^{15,48,49}$. MOTS-c functions 

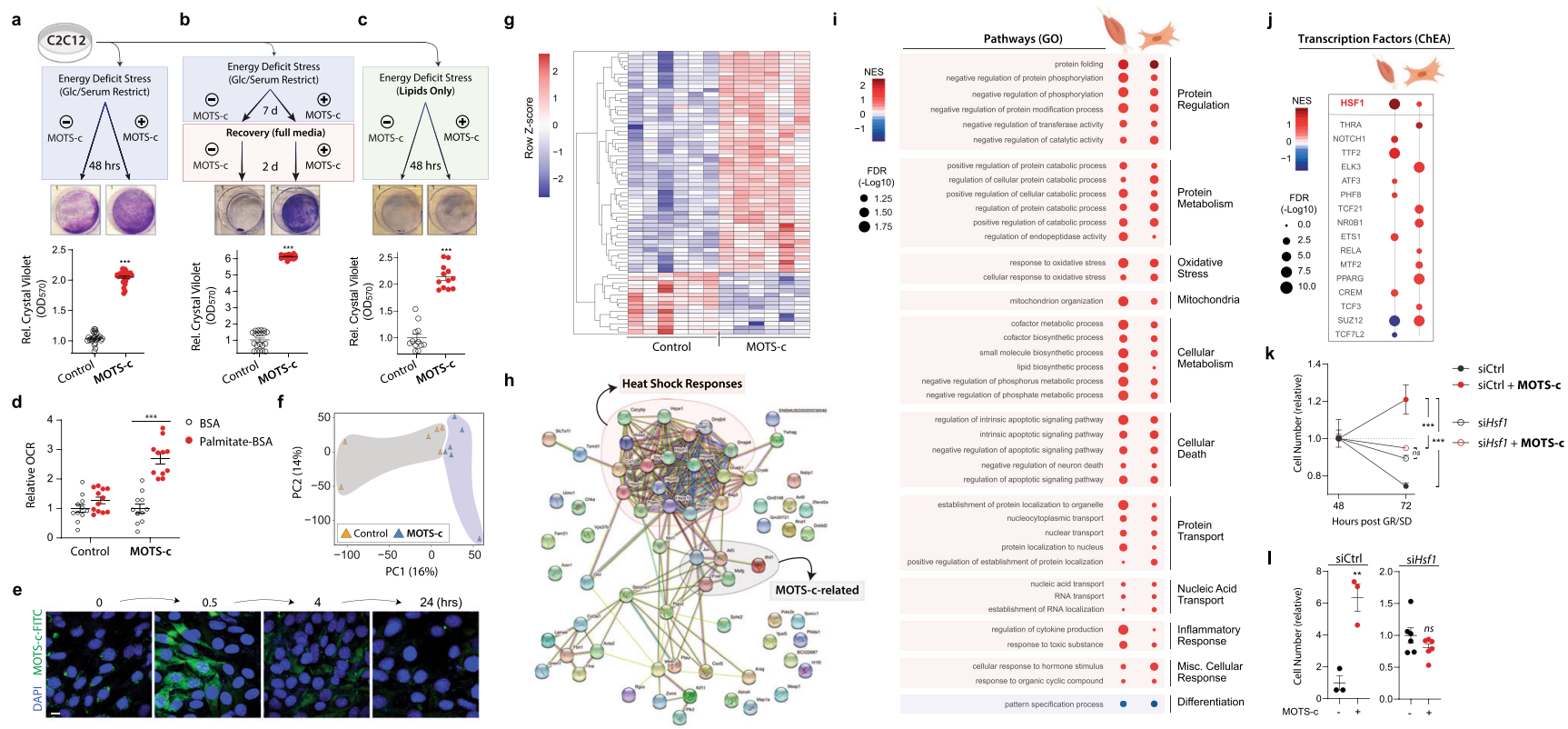

Fig. 5 MOTS-c regulates myoblast gene expression and enhances adaptation to metabolic stress. a-c Survival of MOTS-c-treated (10 $\mu$ M; equal-volume vehicle control) $\mathrm{C} 2 \mathrm{C} 12$ myoblasts assessed by crystal violet staining following a $48 \mathrm{~h}$ of glucose restriction (GR; $0.5 \mathrm{~g} / \mathrm{L}$ ) and serum deprivation (SD; $1 \%$ FBS) with MOTS-c treated only once initially $(n=12$, duplicates; $P=2.57 \mathrm{E}-25)$, $\mathbf{b} 7$ days of GR/SD with daily MOTS-c treatment, followed by a 2-day recovery in full media with MOTS-c $(n=10$, duplicates; $P=8.95 \mathrm{E}-22)$, and $\mathbf{c} 48 \mathrm{~h}$ of complete $\mathrm{GR}(\mathrm{g} / \mathrm{L})$ with chemically defined lipid supplementation and daily MOTS-c treatment ( $n=6$, duplicates; $P=1.17 \mathrm{E}-09$ ). $\mathbf{d}$ Real-time oxygen consumption rate (OCR) in response to fatty acid (palmitate-BSA) in C2C12 myoblasts treated with MOTS-c $(10 \mu \mathrm{M})$ for $48 \mathrm{~h}(n=11$, BSA baseline; $n=12$, palmitate addition; $P=6.27 \mathrm{E}-07)$. e Time-dependent subcellular localization pattern of exogenously treated MOTS-c-FITC $(10 \mu \mathrm{M})$ in C2C12 myoblasts. Scale bar: $10 \mu \mathrm{m}$. Images represent two biological replicates and three technical replicates. f-i RNA-seq was performed on C2C12 myoblasts following $48 \mathrm{~h}$ of GR/SD with/without a unique initial MOTS-c (10 $\mu$ M) treatment $(n=6)$. f Principle Component Analysis (PCA) and $\mathbf{g}$ heatmap of significantly differentially regulated genes by MOTS-c at false discovery rate $(F D R)<5 \%$ by DESeq2 analysis. h Protein-protein interaction network analysis based on genes that were significantly differentially regulated by MOTS-c (FDR $<5 \%$ ) using the STRING (Search Tool for the Retrieval of Interacting Genes/Proteins) database version 11.079. i Balloon plots of common biological processes derived from RNA-seq data between MOTS-c-treated (i) skeletal muscle from old mice (see Fig. 3) and (ii) C2C12 myoblasts, based on gene set enrichment analysis (GSEA) using gene ontology biological process (GO_BP). $\mathbf{j}$ Balloon plots of common transcription factors derived from RNA-seq data between MOTS-c-treated (i) skeletal muscle from old mice (see Fig. 3) and (ii) C2C12 myoblasts, based on ChEA database. $\mathbf{k}$, I Relative cell number at $\mathbf{k}$ 48 vs $72 \mathrm{~h}$ of GR/SD assessed by crystal violet staining $(n=6 ; P=0.00027$, siCtrl + MOTS-c vs. siHsf1 + MOTS-c; $P=0.00017$, siCtrl vs. siCtrl + MOTSc.) and $\mathbf{I} 72 \mathrm{~h}$ of GR/SD assessed by flow cytometry $(n=3$, siCtrl; $n=6$, siHsfl; $P=0.0053)$. Data expressed as mean \pm SEM. Two-sided Student's $t$ test. ${ }^{\star} P<0.05,{ }^{\star \star} P<0.01,{ }^{\star \star \star} P<0.001$.

have been shown to be dependent on the master nutrient sensor AMPK $15,17,34,36,50-54$, including its nuclear translocation in response to metabolic imbalance ${ }^{55}$. Notably, MOTS-c treatment in mice activated AMPK in skeletal muscle and increased the expression of its downstream glucose transporter (i.e GLUT4) ${ }^{15}$, suggesting improved muscle metabolism and physical capacity ${ }^{56}$. Notably, MOTS-c treatment improved running capacity independent of body weight, based on total work (joules) output and lack of correlation between body weight and running time (Supplementary Figs. 4 and 7). This indicates that MOTS-c treatment enhanced physical performance by improving whole body energy metabolism, in part, by promoting adaptive responses to exercise-related stress conditions (e.g. metabolic imbalance and heat shock) in skeletal muscle. Further, the treatment duration of MOTS-c was important to enhance performance (Fig. 2a-h and Supplementary Fig. 5), suggesting the need to reach a certain physiological state with an improved metabolic profile.

MOTS-c acts as an intracellular and endocrine factor, thus has been dubbed a mitochondrial-encoded hormone or a mitochondrial cytokine (mitokine) $2,10,16,49,57-59$. Understanding the detailed molecular details of MOTS-c function as an endocrine factor is an ongoing investigation at multiple ends, including its cellular uptake. The cellular entry of MOTS-c is likely a crucial step in exerting its effects and can occur rapidly, within 30 minutes of treatment
(Fig. 5e $)^{17}$, and appears to be highly regulated in a contextdependent manner (unpublished observation). Notably, antimicrobial peptides (AMPs), which are small amphiphilic and cationic peptides (as small as 15-20 amino acids) like MOTS-c ${ }^{17}$, provide an excellent example of bioactive peptides that can enter cells without being degraded and retainbiological activity ${ }^{60}$; the cellular entry of peptides is still largely unclear and known to adopt a wide range of mechanisms in a concentration-dependent fashion, including energy-dependent transport via endocytosis ${ }^{61,62}$.

Mitochondria are strongly implicated in aging at multiple levels $1,2,24,63-65$ and MOTS-c may contribute to longevity by promoting cellular homeostasis $2,10,57-59$. Here, we present evidence that the mitochondrial genome encodes for instructions to maintain physical capacity (i.e. performance and metabolism) during aging and thereby increase healthspan. MOTS-c treatment initiated in late-life, proximal to the age at which the lifespan curve rapidly descends for $\mathrm{C} 57 \mathrm{BL} / 6 \mathrm{~N}$ mice, significantly delayed the onset of age-related physical disabilities, suggesting "compression of morbidity" in later life ${ }^{66}$. Interestingly, an exceptionally long-lived Japanese population harbors a mitochondrial DNA (mtDNA) SNP $(\mathrm{m} .1382 \mathrm{~A}>\mathrm{C})$ that yields a functional variant of MOTS-c $\mathrm{c}^{67,68}$.

Our study shows that exogenously treated MOTS-c enters the nucleus and regulates nuclear gene expression, including those involved in heat shock response and metabolism. Thus, age- 
related gene networks are comprised of integrated factors encoded by both genomes, which entails a bi-genomic basis for the evolution of aging. Although the detailed molecular mechanism (s) underlying the functions of MOTS-c is an active field of research we provide a proof-of-principlestudy that realizes the mitochondrial genome as a source for instructions that can regulate physical capacity and healthy aging.

\begin{abstract}
Methods
Mouse care. All animal work was approved by and performed in accordance with the University of Southern California (USC) Institutional Animal Care and Use Committee. Mice were housed 3-4 per cage in a facility with a 12:12 h light-dark cycle (light period $0600-1800$ ) at $24^{\circ} \mathrm{C}$. Food and water were available ad libitum. MOTS-c (New England Peptide, USA) was administered daily at 5 or $15 \mathrm{mg} / \mathrm{kg}$ via intraperitoneal injections. In all, 12-week-old male CD-1 (outbred) mice (Charles River, USA), 12-week old male C57BL/6J mice (Jackson Laboratory) and 8- and 18month-old male C57BL/6N mice (National Institute on Aging; NIA) were obtained. All mice were fed either a HFD (60\% calories from fat) or matching control diet (Research Diets, USA, \#D12492 and D12450J, respectively). NIA mice were sufficiently acclimated for 4 months in our vivarium until they were considered middle-aged $(12 \mathrm{mo}$.) and old $(22 \mathrm{mo}$.$) at the start of MOTS-c injections.$ Body weight and food consumption were recorded daily, while body composition was analyzed twice weekly using an LF90II time-domain NMR minispec (Bruker, USA). After eight weeks of injections (23.5 months of age), mice were transitioned to receive MOTS-c injections three times weekly. No live mouse was censored.
\end{abstract}

\section{Physical tests in mice}

Running test. Prior to running training/testing, mice were acclimated to the stationary treadmill apparatus (TSE-Systems, USA) for $10 \mathrm{~min}$ on two consecutive days (Days 1 and 2). Both the high intensity test and training protocols were adapted from published protocols $s^{69,70}$. Running training was given twice on nonconsecutive days and consisted of a fixed speed run of $10 \mathrm{~m} / \mathrm{min}$ for $20 \mathrm{~min}$ (Days 4 and 6) on a level treadmill. The treadmill test on Day 10 consisted of three stages. Stage one was a five-minute run at $13 \mathrm{~m} / \mathrm{min}$. For the next $5 \mathrm{~min}$, the speed was increased by $1 \mathrm{~m} / \mathrm{min}$. The mice run at a fixed speed of $18 \mathrm{~m} / \mathrm{min}$ for the next $30 \mathrm{~min}$. Finally, after $40 \mathrm{~min}$ of total run time, the running speed is increased to $23 \mathrm{~m} / \mathrm{min}$ until exhaustion is reached. All training and testing were done on a level treadmill. Mice resting on the platform were gently prodded to encourage reengagement. Any mouse that resisted prodding and remained on the platform for $30 \mathrm{~s}$ was considered to be exhausted, and time was recorded.

Walking test. When the mice reached 30 months of age, they were no longer capable of performing the same treadmill routine. We developed a measure of mobility in the aged mice consisting of a 60 -s walking test. The treadmill was set at $13 \mathrm{~m} / \mathrm{min}$ for $60 \mathrm{~s}$. We recorded whether the mouse was able to walk, or not, on the treadmill for $60 \mathrm{~s}$, with gentle prodding as needed. Mice remaining on the stationary platform, refusing to engage in the treadmill walking, for more than $5 \mathrm{~s}$ were considered to have failed the test.

Rotarod. The Rotarod test was performed by placing the mice on the apparatus (TSE-Systems), all facing the opposite direction of rotation. The initial speed of rotation was $24 \mathrm{rpm}$ and accelerated at $1 \mathrm{rpm}$ every $10 \mathrm{~s}$. Time to fall was recorded for each mouse, and three trials per mouse was run. Mice received no $<5 \mathrm{~min}$ of recovery time between trials.

Grip strength. We measured grip strength using a horizontal bar connected to the grip strength meter (TSE-Systems) as a high precision force sensor for the forelimbs. After allowing the mouse to properly grip the bar they were firmly and quickly pulled in the opposite direction. Only trials where the mouse released its paws from the bar simultaneously were counted as successful. Mice underwent three trials, with at least $30 \mathrm{~s}$ recovery time between trials.

Gait analysis. To perform gait analysis, we applied a different color of non-toxic ink (BLICK ${ }^{\oplus}$, USA) to the front and hind paw of the mice to record footprints. Barriers were constructed to guide the mice to walk straight on the recording paper. The home cage was kept at the end of the recording paper to encourage completion of the test. Only trials in which the mouse made a continuous, direct path to its home cage were counted. Stride length was measured as the average forward movement of three full strides ${ }^{71}$.

Cognitive tests. Y-maze tests: Mice were placed in a maze consisting of three arms equally spaced $120^{\circ}$ apart. Mice were placed in one arm of the maze and allowed to freely explore the maze for five minutes. Total arm entries and arm choices were recorded for each mouse. An arm entry was defined as a mouse having both front and hind paws entering the arm fully. Percent alternations was defined as an arm choice differing from the previous two compared to the total number of alternation opportunities ${ }^{72}$.
Barnes maze. In all, 12-week-old male CD-1 mice were tested twice daily for 7 days Mice were placed in a start chamber in the middle of the maze and allowed to habituate $(30 \mathrm{~s})$, then the mouse was released to explore the maze and find the escape box (EB). Latency (time to enter the EB) and number of errors (nose pokes and head deflections over false holes) were recorded. A maximum of 2 min was allowed for each trial ${ }^{72}$.

\section{In vivo metabolism assessment}

Metabolic cages. Metabolic activity in mice was measured using the PhenoMaster system (TSE-Systems) equipped to detect indirect calorimetry, measure food and water intake, and monitor activity. Prior to metabolic analysis, mice were housed 3-4 per cage in a facility with a 12:12 h light-dark cycle (light period 0600-1800) at $24^{\circ} \mathrm{C}$. Food and water were available ad libitum. For metabolic assessment, nice were moved into individual PhenoMaster cages in an isolated room under the same environmental conditions. Mice were automatedly monitored for $36 \mathrm{~h}$ to record physiological parameters. To measure $\mathrm{O}_{2}$ intake and $\mathrm{CO}_{2}$ production, gas sensors were calibrated prior to the study using primary gas standards of known concentrations of $\mathrm{O}_{2}, \mathrm{CO}_{2}$, and $\mathrm{N}_{2}$. Room air was passed through the animal chambers at a rate of $0.5 \mathrm{~L} / \mathrm{min}$. Exhaust air from individual cages were sampled at $30-\mathrm{min}$ intervals for $3 \mathrm{~min}$. Sample air was passed through sensors to determine oxygen consumption $\left(\mathrm{VO}_{2}\right)$ and carbon dioxide production $\left(\mathrm{VCO}_{2}\right)$. The respiratory exchange ratio (RER) was calculated as the ratio of carbon dioxide produced to oxygen consumption. The PhenoMaster system allows for activity monitoring using a triple beam IR technology system. Breaking the IR beams through movement was considered a "count". The three-beam system allows XYZ monitoring that considers both ambulatory activity around the cage as well as rearing activity. All data are expressed as the mean of three 24-hour acquisition cycles.

Blood glucose. Blood was collected via a single tail-nick and immediately analyzed using a glucometer (Freestyle, Abbott). Blood collection was performed by trained professionals and in accordance with the University of Southern California Institutional Animal Care and Use Committee.

Western blots. Protein samples were lysed in 1\% Triton X-100 (Thermo Fisher Scientific, USA, \#21568-2500) with $1 \mathrm{mM}$ EDTA (Promega Life Sciences, USA, \#V4231) and $100 \mathrm{mM}$ Tris-HCl pH 7.5 (Quality Biological, USA, \#351-006-101) and protease inhibitors (Roche, Germany, \#118636170001) and sonicated using a Sonic Dismembrator (Fisher Scientific, USA). Samples were heated at $95^{\circ} \mathrm{C}$ for 5 min. Samples were ran on 4-20\% gradient tris-glycine gels (TGX; Bio-Rad, USA, \#456-1104) and transferred onto 0.2 $\mu \mathrm{M}$ PVDF membranes (Bio-Rad \#162-0184) using a Transblot Turbo semi-dry transfer system (Bio-Rad) at 9 volts for $15 \mathrm{~min}$ Membranes were blocked for $1 \mathrm{~h}$ using 5\% BSA (Akron Biotech, USA, \#AK89050100) in tris-buffered saline containing 0.05\% Tween-20 (Bio-Rad \#161-0781) and incubated in primary antibodies against MOTS-c (1:250, rabbit polyclonal; YenZym, USA) and GAPDH (1:1,000, cat\# 5174; Cell Signaling, USA) overnight at $4^{\circ} \mathrm{C}$. Secondary HRP-conjugated antibodies (\#7074; Cell Signaling, USA) were then added $(1: 30,000)$ for one hour at room temperature. Chemiluminescence was detected and imaged using Clarity western ECL substrate (Bio-Rad \#1705060) and Chemidoc XRS system (Bio-Rad). Western blots were quantified using ImageJ version $1.52 \mathrm{k}$.

\section{Cell studies}

Cell culture. C2C12 cells were cultured in DMEM with $4.5 \mathrm{~g} / \mathrm{L}$ glucose (Corning, USA \#10-017-CV) and 10\% FBS (Millipore-Sigma, USA, \#F0926-500). All cells were stored at $37^{\circ} \mathrm{C}$ and $5 \% \mathrm{CO}_{2}$. Cells were passaged when they reached $75-80 \%$ confluence using TrypLE (Thermo Fisher Scientific \#12605-010).

Cell survival assays. Protection against glucose restriction (GR) and serum deprivation (SD) was tested by culturing cells in DMEM (Thermo Fisher Scientific \#11966-025) with 0.5 g/L glucose (Millipore-Sigma \#G8769) and 1\% FBS. MOTS-c $(10 \mu \mathrm{M})$ or vehicle (PBS). MOTS-c $(10 \mu \mathrm{M})$ was added to the media every $24 \mathrm{~h}$. After $48 \mathrm{~h}$ of GR/SD, we performed crystal violet (Thermo Fisher Scientific \#C58125) staining as before ${ }^{17}$ to determine cell survival. We also tested cellular proliferation, following prolonged (7-day) GR/SD (DMEM with $1 \% \mathrm{FBS}$ and $0.5 \mathrm{~g} / \mathrm{L}$ glucose), as a measure of cellular fitness. In this case, MOTS-c-containing $(10 \mu \mathrm{M})$ media was changed once every 2 days; no additional MOTS-c supplementation was given between media changes. After 7 days of GR/SD, we returned the cells to full growth media (10\% FBS and $4.5 \mathrm{~g} / \mathrm{L}$ glucose) for $48 \mathrm{~h}$ with MOTS-c $(10 \mu \mathrm{M})$, then stained them with crystal violet. To determine the metabolic flexibility to utilize fatty acids, we cultured cells in DMEM with $1 \%$ FBS, $0.5 \mathrm{~g} / \mathrm{L}$ glucose, and $1 \%$ chemically defined lipid mixture (Millipore-Sigma \#L0288) for $48 \mathrm{~h}$, then stained them with crystal violet.

Metabolic flux. Real-time oxygen consumption and extracellular acidification rates in $\mathrm{C} 2 \mathrm{C} 12$ myoblasts treated with $16 \%$ palmitate-BSA ( $1 \mathrm{mM}$ palmitate conjugated to $0.17 \mathrm{mM} \mathrm{BSA})$ or $16 \% \mathrm{BSA}(0.17 \mathrm{mM}$; Seahorse Bioscience \#102720-100) were obtained using the XF96 Bioanalyzer (Seahorse Bioscience) at the USC Leonard Davis School of Gerontology Seahorse Core. All values were normalized to relative 
protein concentration using a BCA protein assay kit (Thermo Fisher Scientific \#23227).

Confocal microscopy. Confocal images were obtained using a Zeiss Confocal Laser Scanning Microscope 700 (Zeiss, Germany). C2C12 myoblasts were cultured on glass coverslips (Chemglass, USA, \#CLS-1760-015). Cells were treated with FITCMOTS-c (New England Peptide) for either $0 \mathrm{~h}$ (immediate), $30 \mathrm{~min}, 4 \mathrm{~h}$, or $24 \mathrm{~h}$. All cells were treated with Hoeschst (Biotium, USA, \#40045) for $15 \mathrm{~min}$ and then washed three times with PBS. Cells were fixed in 10\% formalin (Millipore-Sigma \#EM-R04586-82) and washed an additional three times in PBS. Coverslips were affixed to glass slides (VWR, USA, \#48300-025) using ProLong Gold antifade reagent (Life Technologies Corporation, USA, \#P36934).

\section{Human studies}

Study outline. Participants gave written consent before the commencement of the study, which was approved by the Northern Health and Disability Ethics Committee, and carried out in accordance with guidelines for human research (New Zealand; 16/STH/116/AM01). In total, 10 sedentary ( $<4 \mathrm{~h}$ aerobic exercise/week) healthy young males $(24.5 \pm 3.7$ years old and BMI $24.1 \pm 2.1)$ were recruited to take part in a two-visit exercise trial. Recruited participants were free of cardiovascular, metabolic and blood diseases and were not taking any medication or supplements. The trial was separated into two visits, each involving exercise bouts that were carried out on an electromagnetically braked cycle ergometer (Velotron, RacerMate, USA).

Determination of peak oxygen uptake $\left(\mathrm{VO}_{2}\right.$ peak) and maximal power output (visit 1). Peak oxygen uptake was determined using a ramped cycling exercise protocol. Prior to testing, participants warmed up for five minutes at a self-selected workload between 60 and $80 \mathrm{~W}$. The ramp protocol began at $60 \mathrm{~W}$, with the cycling power output set to increase by $1 \mathrm{~W}$ every $4 \mathrm{~s}(15 \mathrm{~W} / \mathrm{min})$ continuously until the participant was unable to maintain cycling workload (cycling cadence $<$ minimum 60 revolutions per minute) or maximal volitional fatigue was reached. Mean Peak oxygen uptake of participants was reported as $38.4 \pm 7.3 \mathrm{ml} . \mathrm{kg} . \mathrm{min}$.

Acute high intensity cycling exercise session (visit 2). Prior to visit 2, participants were asked to fast overnight (from 10PM) and were instructed to abstain from physical activity for at least $48 \mathrm{~h}$ prior. Upon arrival to the laboratory, participants lay supine for 15-min and then had an intravenous cannula inserted into a forearm vein. A resting plasma sample was collected followed by a pre-exercise muscle biopsy taken from the vastus lateralis muscle (quadriceps muscle). Approximately 10-min later, participants completed 10, 60-s cycling intervals at individuallyspecified peak power workloads (determined from peak oxygen uptake test) followed by 75 -seconds of rest/low intensity cycling $(<30 \mathrm{~W})$ per interval ${ }^{73}$. A midexercise blood sample was taken following the completion of the 5th exercise interval as well as immediately following the completion of the exercise bout. In addition, an immediately-post exercise muscle biopsy was taken (within $\sim 5$-min of completion of the exercise bout). Participants remained supine and resting in the procedure bed for a 4 -h recovery period. Following 4 -h of recovery, a final blood and muscle biopsy sample was collected.

Muscle biopsy and blood sampling. Muscle biopsies were extracted under local anesthesia (1\% xylocaine) using the Bergstrom needle with manual suction technique $^{74}$. Biopsies were snap-frozen in liquid nitrogen and stored at $-80{ }^{\circ} \mathrm{C}$ until analyzed. Blood was drawn through a 20 -gauge cannula, collected in 10-mL EDTA vacutainers and then centrifuged immediately upon collection at $4^{\circ} \mathrm{C}$ at $2000 \times g$ for $10 \mathrm{~min}$. Plasma was extracted and then stored at $-80^{\circ} \mathrm{C}$ until further analysis using an in-house ELISA as described before ${ }^{15}$. Human skeletal muscle was processed for western blotting by soaking the samples in lysis buffer (above) and minced using a razor blade. Once the sample was evenly minced, we proceeded with the Sonic Dismembrator step as described above.

\section{Liquid chromatography-mass spectrometry metabolomics. Metabolites were} extracted from randomly selected tissue samples by adding $1 \mathrm{~mL}$ of 80:20 methanol:water solution on dry ice. Samples were incubated at $-80^{\circ} \mathrm{C}$ for $4 \mathrm{~h}$ and centrifuged at $4^{\circ} \mathrm{C}$ for $5 \mathrm{~min}$ at $21,000 \times \mathrm{g}$. Supernatants were transferred into LoBind Eppendorf microcentrifuge tubes and the cell pellets were re-extracted with $200 \mu \mathrm{L}$ ice-cold $80 \% \mathrm{MeOH}$, spun down and the supernatants were combined. Metabolites were dried at room temperature under vacuum and resuspended in water for injection.

Samples were randomized and analyzed on a Q-Exactive Plus hybrid quadrupole-Orbitrap mass spectrometer coupled to an UltiMate 3000 UHPLC system (Thermo Scientific). The mass spectrometer was run in polarity switching mode $(+3.00 \mathrm{kV} /-2.25 \mathrm{kV})$ with an $\mathrm{m} / \mathrm{z}$ window ranging from 65 to 975 . Mobile phase A was $5 \mathrm{mM}$ NH4AcO, pH 9.9, and mobile phase B was acetonitrile. Metabolites were separated on a Luna $3 \mu \mathrm{m} \mathrm{NH} 2100 \AA(150 \times 2.0 \mathrm{~mm})$ column (Phenomenex). The flowrate was $300 \mu \mathrm{l} / \mathrm{min}$, and the gradient was from $15 \% \mathrm{~A}$ to $95 \% \mathrm{~A}$ in $18 \mathrm{~min}$, followed by an isocratic step for $9 \mathrm{~min}$ and re-equilibration for $7 \mathrm{~min}$. All samples were injected twice for technical duplicates. Metabolites were detected and quantified as area under the curve based on retention time and accurate mass $(\leq 5 \mathrm{ppm})$ using the TraceFinder 3.3 (Thermo Scientific) software.

\section{RNA-seq}

RNA purification from tissue and cells. Total RNA extraction from skelatal muscle tissue or $\mathrm{C} 2 \mathrm{C} 12$ mouse myoblasts was done using TRI Reagent (Millipore-Sigma \#T9424). Muscle tissue samples were flash-frozen in liquid nitrogen until further processing. Tissues were resuspended in $600 \mu \mathrm{L}$ of TRI Reagent, then homogenized on Lysing Matrix D $2 \mathrm{~mL}$ tubes (MP Biomedicals) on a BeadBug homogenizer (Benchmark Scientific). For both skeletal muscle and C2C12 cells, total RNA was purified using the Direct-zol RNA MiniPrep (Zymo Research \#R2052).

RNA-seq library preparation. Total RNA was subjected to rRNA depletion using the NEBNext rRNA Depletion Kit (New England Biolabs), according to the manufacturer's protocol. Strand specific RNA-seq libraries were then constructed using the SMARTer Stranded RNA-Seq Kit (Clontech \# 634839), according to the manufacturer's protocol. Based on rRNA-depleted input amount, 13-15 cycles of amplification were performed to generate RNA-seq libraries. Paired-end $150 \mathrm{bp}$ reads were sent for sequencing on the Illumina HiSeq-Xten platform at the Novogene Corporation (USA). The raw sequencing data was deposited to the NCBI Sequence Read Archive (accession: PRJNA556045). The resulting data was then analyzed with a standardized RNA-seq data analysis pipeline (described below).

RNA-seq analysis pipeline. To avoid the mapping issues due to overlapping sequence segments in paired end reads, reads were hard trimmed to $75 \mathrm{bp}$ using the Fastx toolkit v0.0.13. Reads were then further quality-trimmed using Trimgalore 0.4.4 (github.com/FelixKrueger/TrimGalore) to retain high-quality bases with Phred score $>20$. All reads were also trimmed by $6 \mathrm{bp}$ from their $5^{\prime}$ end to avoid poor qualities or biases. cDNA sequences of protein coding and lincRNA genes were obtained through ENSEMBL Biomart for the GRCm 38 build of the mouse genome (Ensemble release v94). Trimmed reads were mapped to this reference using kallisto $0.43 .0-1$ and the $-\mathrm{fr}$-stranded option ${ }^{75}$. All subsequent analyses were performed in the $\mathrm{R}$ statistical software (https://cran.r-project.org/).

Read counts were imported into $\mathrm{R}$, and summarized at the gene level, to estimate differential gene expression as a function of age.

Because of high sample variability, we used surrogate variable analysis to remove experimental noise from the muscle RNA-seq dataset ${ }^{76}$. R package 'sva' v3.24.4 $4^{77}$ was used to estimate surrogate variable, and the effects of surrogate variables were regressed out using 'limma'. Corrected read counts were then used for downstream analyses.

DEseq2 normalized fold-changes were then used to estimate differential gene expression between control and MOTS-c treated muscle or cell samples using the 'DESeq2' R package (DESeq2 1.16.1) ${ }^{78}$. The heatmap of expression across samples for significant genes (Fig. $5 \mathrm{~g}$ ) was plotted using the R package 'pheatmap' 1.0.10 (Raivo Kolde, 2015-12-11; https://CRAN.R-project.org/package=pheatmap). Putative protein-protein interaction was derived using the STRING (Search Tool for the Retrieval of Interacting Genes/Proteins) database version $11.0^{79}$ (https:// string-db.org/)

Functional enrichment analysis. To perform functional enrichment analysis, we used the Gene Set Enrichment Analysis [GSEA] paradigm through its implementation in the R package 'ClusterProfiler' v3.10.1 ${ }^{80}$, and Bioconductor annotation package 'org.Mm.eg.db' v3.7.0. Balloon plots representing the output were generated using R packages 'ggplot2' v3.1.0 and 'scales' 1.0.0. GSEA analysis was conducted using the Genome Ontology information from the R 'org.Mm.eg.db' package, as well as the ChEA knowledge base derived from EnrichR ${ }^{43}$, as formatted in our previous study ${ }^{81}$.

\section{Principal component analysis}

Metabolites. Principal component analysis (PCA) was performed using the meancentered matrix of metabolite values per each mouse. Principal components that separated sample groups were identified with visual inspection. Loadings from principal components that stratify experimental samples versus controls were then queried against metabolic pathways using a Kolmogorov-Smirnov statistic against the expected distribution of metabolites. Metabolic pathway enrichment analysis (gene set enrichment analysis, GSEA) ${ }^{82}$ were performed using 28 metabolic pathways defined by the Kyoto Encyclopedia of Genes and Genomes (KEGG) database using pathways with four or more measured metabolites.

RNA-seq. PCA was performed using the $\mathrm{R}$ base package function 'prcomp'. The first 2 principal components were used.

Quantification and statistical analysis. Statistical significance was determined using the two-sided Student's $t$ test. Statistical tests were performed using GraphPad Prism version 8.1.2. Results of $t$-tests are indicated in all figures as $* P<$ $0.05, * * P<0.01, * * * P<0.001$ and ns for not significant $(p>0.05)$. 
The RNA-seq analytical code will be made available on the Benayoun lab github (https://github.com/BenayounLaboratory/MOTSc_Exercise).

Reporting summary. Further information on research design is available in the Nature Research Reporting Summary linked to this article.

\section{Data availability}

All data are available in the main manuscript and supplementary material and all relevant data is available from the authors. RNA-seq data have been uploaded to the NCBI SRA database accession: PRJNA556045 https://doi.org/10.5281/zenodo.4267090. A Source Data file is available for this article. Source data are provided with this paper.

\section{Code availability:}

The RNA-seq analytical code will be made available on the Benayoun lab github (https:// github.com/BenayounLaboratory/MOTSc_Exercise). Source data are provided with this paper.

Received: 24 December 2019; Accepted: 18 December 2020; Published online: 20 January 2021

\section{References}

1. Lopez-Otin, C., Blasco, M. A., Partridge, L., Serrano, M. \& Kroemer, G. The hallmarks of aging. Cell 153, 1194-1217 (2013).

2. Lopez-Otin, C., Galluzzi, L., Freije, J. M., Madeo, F. \& Kroemer, G. Metabolic control of longevity. Cell 166, 802-821 (2016).

3. Kaeberlein, M., Rabinovitch, P. S. \& Martin, G. M. Healthy aging: the ultimate preventative medicine. Science 350, 1191-1193 (2015).

4. Campisi, J. et al. From discoveries in ageing research to therapeutics for healthy ageing. Nature 571, 183-192 (2019).

5. Kennedy, B. K. et al. Geroscience: linking aging to chronic disease. Cell 159, 709-713 (2014).

6. De Magalhães, J. P., Stevens, M. \& Thornton, D. The business of anti-aging science. Trends Biotechnol. 35, 1062-1073 (2017).

7. Dzau, V. J., Inouye, S. K., Rowe, J. W., Finkelman, E. \& Yamada, T. Enabling healthful aging for all - the national academy of medicine grand challenge in healthy longevity. New Engl. J. Med. https://doi.org/10.1056/nejmp1912298 (2019).

8. Barzilai, N., Cuervo, A. M. \& Austad, S. Aging as a biological target for prevention and therapy. JAMA 320, 1321 (2018).

9. Olshansky, S. J. From lifespan to healthspan. JAMA 320, 1323 (2018)

10. Quirós, P. M., Mottis, A. \& Auwerx, J. Mitonuclear communication in homeostasis and stress. Nat. Rev. Mol. Cell Biol. 17, 213-226 (2016).

11. Gottschling, D. E. \& Nyström, T. The upsides and downsides of organelle interconnectivity. Cell 169, 24-34 (2017).

12. Matilainen, O., Quirós, P. M. \& Auwerx, J. Mitochondria and epigenetics crosstalk in homeostasis and stress. Trends Cell Biol. 27, 453-463 (2017).

13. Kim, S.-J., Xiao, J., Wan, J., Cohen, P. \& Yen, K. Mitochondrially derived peptides as novel regulators of metabolism. J. Physiol. 595, 6613-6621 (2017).

14. Lee, C., Yen, K. \& Cohen, P. Humanin: a harbinger of mitochondrial-derived peptides? Trends Endocrinol. Metab. https://doi.org/10.1016/j.tem.2013.01.005 (2013).

15. Lee, C. et al. The mitochondrial-derived peptide MOTS-c promotes metabolic homeostasis and reduces obesity and insulin resistance. Cell Metab. 21, 443-454 (2015).

16. Zarse, K. \& Ristow, M. A mitochondrially encoded hormone ameliorates obesity and insulin resistance. Cell Metab. 21, 355-356 (2015).

17. Kim, K. H., Son, J. M., Benayoun, B. A. \& Lee, C. The mitochondrial-encoded peptide mots-c translocates to the nucleus to regulate nuclear gene expression in response to metabolic stress. Cell Metab. 28, 516-524 e517 (2018).

18. Mangalhara, K. C. \& Shadel, G. S. A mitochondrial-derived peptide exercises the nuclear option. Cell Metab. 28, 330-331 (2018).

19. Ramanjaneya, M. et al. Mitochondrial-derived peptides are down regulated in diabetes subjects. Front. Endocrinol. https://doi.org/10.3389/fendo.2019.00331 (2019).

20. Yong, C. Q. Y. \& Tang, B. L. A Mitochondrial encoded messenger at the nucleus. Cells https://doi.org/10.3390/cells7080105 (2018)

21. Alis, R., Lucia, A., Blesa, J. R. \& Sanchis-Gomar, F. The role of mitochondrial derived peptides (MDPs) in metabolism. J. Cell. Physiol. 230, 2903-2904 (2015).

22. Li, S. \& Laher, I. Exercise pills: at the starting line. Trends Pharmacol. Sci. 36, 906-917 (2015).

23. Knoop, A., Thomas, A. \& Thevis, M. Development of a mass spectrometry based detection method for the mitochondrion-derived peptide MOTS-c in plasma samples for doping control purposes. Rapid Commun. Mass Spectrom. 33, 371-380 (2019).

24. Jang, J. Y., Blum, A., Liu, J. \& Finkel, T. The role of mitochondria in aging. J. Clin. Investig. 128, 3662-3670 (2018).

25. Goodpaster, B. H. \& Sparks, L. M. Metabolic flexibility in health and disease. Cell Metab. 25, 1027-1036 (2017).

26. Smith, R. L., Soeters, M. R., Wüst, R. C. I. \& Houtkooper, R. H. Metabolic flexibility as an adaptation to energy resources and requirements in health and disease. Endocr. Rev. 39, 489-517 (2018).

27. Harrison, D. E. et al. Rapamycin fed late in life extends lifespan in genetically heterogeneous mice. Nature 460, 392-395 (2009).

28. Mao, K. et al. Late-life targeting of the IGF-1 receptor improves healthspan and lifespan in female mice. Nat. Commun. https://doi.org/10.1038/s41467018-04805-5 (2018).

29. Rae, M. J. et al. The demographic and biomedical case for late-life interventions in aging. Sci. Transl. Med. 2, 40 cm21-40 cm21 (2010).

30. Neufer, P. D. et al. Understanding the cellular and molecular mechanisms of physical activity-induced health benefits. Cell Metab. 22, 4-11 (2015).

31. Cataldo, L. R., Fernandez-Verdejo, R., Santos, J. L. \& Galgani, J. E. Plasma MOTS-c levels are associated with insulin sensitivity in lean but not in obese individuals. J. Investig. Med. 66, 1019-1022 (2018).

32. Du, C. et al. Circulating MOTS-c levels are decreased in obese male children and adolescents and associated with insulin resistance. Pediatr. Diabetes https://doi.org/10.1111/pedi.12685 (2018).

33. $\mathrm{Li}, \mathrm{Q}$. et al. Earlier changes in mice after D-galactose treatment were improved by mitochondria derived small peptide MOTS-c. Biochem. Biophys. Res. Commun. https://doi.org/10.1016/j.bbrc.2019.03.194 (2019).

34. Lu, H. et al. MOTS-c peptide regulates adipose homeostasis to prevent ovariectomy-induced metabolic dysfunction. J. Mol. Med. https://doi.org/ 10.1007/s00109-018-01738-w (2019).

35. Ramanjaneya, M. et al. Lipids and insulin regulate mitochondrial-derived peptide (MOTS-c) in PCOS and healthy subjects. Clin. Endocrinol. https://doi. org/10.1111/cen.14007 (2019).

36. Lu et al. Mitochondrial-derived peptide MOTS-c increases adipose thermogenic activation to promote cold adaptation. Int. J. Mol. Sci. 20, 2456 (2019).

37. Hawley, J. A., Hargreaves, M., Joyner, M. J. \& Zierath, J. R. Integrative biology of exercise. Cell 159, 738-749 (2014).

38. Egan, B. \& Juleen Exercise metabolism and the molecular regulation of skeletal muscle adaptation. Cell Metab. 17, 162-184 (2013).

39. Packer, L., Cadenas, E. \& Davies, K. J. A. Free radicals and exercise: an introduction. Free Radic. Biol. Med. 44, 123-125 (2008).

40. Merry, T. L. \& Ristow, M. Do antioxidant supplements interfere with skeletal muscle adaptation to exercise training? J. Physiol. https://doi.org/10.1113/ jp270654 (2015)

41. Chrétien, D. et al. Mitochondria are physiologically maintained at close to $50^{\circ}$ C. PLoS Biol. 16, e2003992 (2018)

42. Kim, S. J. et al. The mitochondrial-derived peptide MOTS-c is a regulator of plasma metabolites and enhances insulin sensitivity. Physiol. Rep. https://doi. org/10.14814/phy2.14171 (2019).

43. Kuleshov, M. V. et al. Enrichr: a comprehensive gene set enrichment analysis web server 2016 update. Nucleic Acids Res. 44, W90-W97 (2016).

44. Li, J., Labbadia, J. \& Morimoto, R. I. Rethinking HSF1 in stress, development, and organismal health. Trends Cell Biol. 27, 895-905 (2017).

45. Anckar, J. \& Sistonen, L. Regulation of HSF1 function in the heat stress response: implications in aging and disease. Annu. Rev. Biochem. 80 1089-1115 (2011)

46. Vasilaki, A., McArdle, F., Iwanejko, L. M. \& McArdle, A. Adaptive responses of mouse skeletal muscle to contractile activity: the effect of age. Mech. Ageing Dev. 127, 830-839 (2006).

47. Sakamoto, M. et al. Upregulation of heat shock transcription factor 1 plays a critical role in adaptive cardiac hypertrophy. Circ. Res. 99, 1411-1418 (2006).

48. Hesselink, M. K. C., Schrauwen-Hinderling, V. \& Schrauwen, P. Skeletal muscle mitochondria as a target to prevent or treat type 2 diabetes mellitus. Nat. Rev. Endocrinol. 12, 633-645 (2016).

49. Priest, C. \& Tontonoz, P. Inter-organ cross-talk in metabolic syndrome. Nat Metab. 1, 1177-1188 (2019).

50. Ming, W. et al. Mitochondria related peptide MOTS-c suppresses ovariectomy-induced bone loss via AMPK activation. Biochem. Biophys. Res. Commun. 476, 412-419 (2016).

51. Yin, X. et al. The intraperitoneal administration of MOTS-c produces antinociceptive and anti-inflammatory effects through the activation of AMPK pathway in the mouse formalin test. Eur. J. Pharmacol. 870, 172909 (2020).

52. Xinqiang, Y., Quan, C., Yuanyuan, J. \& Hanmei, X. Protective effect of MOTS $c$ on acute lung injury induced by lipopolysaccharide in mice. Int. Immunopharmacol. 80, 106174 (2020).

53. Wei, M. et al. Mitochondrial-derived peptide MOTS-c attenuates vascular calcification and secondary myocardial remodeling via adenosine 
monophosphate-activated protein kinase signaling pathway. Cardiorenal. Med. 10, 42-50 (2020).

54. Yan, Z. et al. MOTS-c inhibits Osteolysis in the Mouse Calvaria by affecting osteocyte-osteoclast crosstalk and inhibiting inflammation. Pharmacol. Res. 147, 104381 (2019).

55. Kim, K. H., Son, J. M., Benayoun, B. A. \& Lee, C. The mitochondrial-encoded peptide MOTS-c translocates to the nucleus to regulate nuclear gene expression in response to metabolic stress. Cell Metab. https://doi.org/10.1016/ j.cmet.2018.06.008 (2018)

56. Li, S. \& Laher, I. Exercise pills: at the starting line. Trends Pharmacol. Sci. 36, 906-917 (2015).

57. Galluzzi, L., Yamazaki, T. \& Kroemer, G. Linking cellular stress responses to systemic homeostasis. Nat. Rev. Mol. Cell Biol. 19, 731-745 (2018).

58. Mottis, A., Herzig, S. \& Auwerx, J. Mitocellular communication: Shaping health and disease. Science 366, 827-832 (2019).

59. Tan, J. X. \& Finkel, T. Mitochondria as intracellular signaling platforms in health and disease. J. Cell Biol. https://doi.org/10.1083/jcb.202002179 (2020).

60. Mookherjee, N., Anderson, M. A., Haagsman, H. P. \& Davidson, D. J. Antimicrobial host defence peptides: functions and clinical potential. Nat. Rev. Drug Discov. https://doi.org/10.1038/s41573-019-0058-8 (2020).

61. Kauffman, W. B., Guha, S. \& Wimley, W. C. Synthetic molecular evolution of hybrid cell penetrating peptides. Nat. Commun. https://doi.org/10.1038/ s41467-018-04874-6 (2018).

62. Ruseska, I. \& Zimmer, A. Internalization mechanisms of cell-penetrating peptides. Beilstein J. Nanotechnol. 11, 101-123 (2020).

63. Wang, Y. \& Hekimi, S. Mitochondrial dysfunction and longevity in animals: untangling the knot. Science 350, 1204-1207 (2015).

64. Kauppila, T. E. S., Kauppila, J. H. K. \& Larsson, N.-G. Mammalian mitochondria and aging: an update. Cell Metab. 25, 57-71 (2017).

65. Son, J. M. \& Lee, C. Mitochondria: multifaceted regulators of aging. BMB Rep. 52, 13-23 (2019).

66. Crimmins, E. M. Lifespan and healthspan: past, present, and promise. Gerontologist 55, 901-911 (2015).

67. Zempo, H. et al. Relation between type 2 diabetes and m. $1382 \mathrm{~A}>\mathrm{C}$ polymorphism which occurs amino acid replacement (K14Q) of mitochondria-derived MOTS-c. FASEB J. 30, 956.951-956.951 (2016).

68. Fuku, N. et al. The mitochondrial-derived peptide MOTS-c: a player in exceptional longevity? Aging Cell 14, 921-923 (2015).

69. Das, A. et al. Impairment of an endothelial NAD $(+)-\mathrm{H} 2 \mathrm{~S}$ signaling network is a reversible cause of vascular aging. Cell 173, 74-89 e20 (2018).

70. Reynolds, J. C. \& Lee, C. Mouse fitness as determined through treadmill running and walking. Methods Mol. Biol. 2144, 57-65 (2020).

71. Carter, R. J., Morton, J. \& Dunnett, S. B. Motor coordination and balance in rodents. Curr. Protoc. Neurosci. https://doi.org/10.1002/0471142301. ns0812s15 (2001).

72. Brandhorst, S. et al. A periodic diet that mimics fasting promotes multi-system regeneration, enhanced cognitive performance, and healthspan. Cell Metab. 22, 86-99 (2015).

73. Hedges, C. P. et al. Peripheral blood mononuclear cells do not reflect skeletal muscle mitochondrial function or adaptation to high-intensity interval training in healthy young men. J. Appl Physiol. 126, 454-461 (2019).

74. Bergstrom, J. Percutaneous needle biopsy of skeletal muscle in physiological and clinical research. Scand. J. Clin. Lab Invest. 35, 609-616 (1975).

75. Bray, N. L., Pimentel, H., Melsted, P. \& Pachter, L. Near-optimal probabilistic RNA-seq quantification. Nat. Biotechnol. 34, 525-527 (2016).

76. Leek, J. T. \& Storey, J. D. Capturing heterogeneity in gene expression studies by surrogate variable analysis. PLoS Genet. 3, e161 (2007).

77. Leek, J. T. et al. sva: Surrogate Variable Analysis. R package version 3.32.1. (2019).

78. Love, M. I., Huber, W. \& Anders, S. Moderated estimation of fold change and dispersion for RNA-seq data with DESeq2. Genome Biol. https://doi.org/ 10.1186/s13059-014-0550-8 (2014).

79. Szklarczyk, D. et al. STRING v11: protein-protein association networks with increased coverage, supporting functional discovery in genome-wide experimental datasets. Nucleic Acids Res. 47, D607-D613 (2019).
80. Yu, G., Wang, L.-G., Han, Y. \& He, Q.-Y. clusterProfiler: an R Package for comparing biological themes among gene clusters. OMICS 16, 284-287 (2012).

81. Benayoun, B. A. et al. Remodeling of epigenome and transcriptome landscapes with aging in mice reveals widespread induction of inflammatory responses. Genome Res. https://doi.org/10.1101/gr.240093.118 (2019).

82. Subramanian, A. et al. Gene set enrichment analysis: A knowledge-based approach for interpreting genome-wide expression profiles. Proc. Natl Acad. Sci. USA 102, 15545-15550 (2005).

\section{Acknowledgements}

We thank the USC Leonard Davis School of Gerontology Mouse Phenotyping core and Seahorse Bioanalyzer core and the USC Genomics core for experimental assistance. Funding was provided by the American Federation for Aging Research (AFAR), the National Institute on Aging (T32 AG052374), and the USC Manning Endowed Fellowship to J.C.R., a Mork Graduate Fellowship from the USC Viterbi School of Engineering to J.H.J., the USC Viterbi School of Engineering to N.A.G., the NIA (P01AG034906) to P.C., the NIA (R00AG049934), an innovator grant from the Rose Hills foundation, a seed grant the NAVIGAGE foundation, and the Hanson-Thorell Family to B.A.B., Rutherford Discovery Fellowship and a Marsden Fund Fast Start Grant to T.L.M., and the NIA (R01AG052258), Ellison Medical Foundation (EMF), AFAR, and the Hanson-Thorell Family to C.L.

\section{Author contributions}

J.C.R., T.L.M., B.A.B., P.C., N.A.G., D.C-S., and C.L. conceived the experiments. J.R. J.S.T.W., B.A.B., R.L., J.H.J., C.J.M., and D.C-S. performed experiments. J.R., T.L.M., J.S.T.W., B.A.B., R.L., P.C., N.A.G., J.H.J., and C.L. analyzed the data. J.R. and C.L. wrote the manuscript. All authors approved the manuscript.

\section{Competing interests}

P.C. and C.L. are consultants and shareholders of CohBar, Inc. All other authors declare no competing interests.

\section{Additional information}

Supplementary information is available for this paper at https://doi.org/10.1038/s41467 020-20790-0.

Correspondence and requests for materials should be addressed to C.L.

Peer review information Nature Communications thanks Julian Griffin and the other, anonymous, reviewer(s) for their contribution to the peer review of this work.

Reprints and permission information is available at http://www.nature.com/reprints

Publisher's note Springer Nature remains neutral with regard to jurisdictional claims in published maps and institutional affiliations.

Open Access This article is licensed under a Creative Commons Attribution 4.0 International License, which permits use, sharing, adaptation, distribution and reproduction in any medium or format, as long as you give appropriate credit to the original author(s) and the source, provide a link to the Creative Commons license, and indicate if changes were made. The images or other third party material in this article are included in the article's Creative Commons license, unless indicated otherwise in a credit line to the material. If material is not included in the article's Creative Commons license and your intended use is not permitted by statutory regulation or exceeds the permitted use, you will need to obtain permission directly from the copyright holder. To view a copy of this license, visit http://creativecommons.org/ licenses/by/4.0/.

(C) The Author(s) 2021 